\title{
A importância do hemograma no diagnóstico precoce da leucemia
}

\author{
The importance of blood count in the early diagnosis of leucemia \\ La importancia del recuento sanguíneo en el diagnóstico precoz de leucemia
}

Robson Azevedo Dutra ${ }^{1 *}$, Camila Aparecida Abrahão ${ }^{1}$, Flávia Mendes Lopes ${ }^{1}$, Rafael Fernando Souza Rocha ${ }^{1}$, Siderleu Pires Rosa Junior ${ }^{1}$.

\section{RESUMO}

Objetivo: Analisar os sinais, sintomas e os valores do hemograma inicial de crianças que tiveram posteriormente o diagnóstico definitivo, por meio do mielograma, de leucemia. Métodos: Foram avaliados prontuários de 31 indivíduos, de ambos os sexos, idade inferior a 18 anos, com diagnóstico de leucemia linfoide e mieloide no serviço de Oncologia de um hospital em uma Cidade do Estado de São Paulo. Foram avaliadas as frequências absolutas e relativas, média, desvio padrão, valores mínimos, máximos e mediana. O estudo foi aprovado por Comitê de Ética em Pesquisa. Resultados: Considerando as variáveis dos hemogramas dos pacientes analisados, observa-se que $80,64 \%$ apresentavam anemia no momento do diagnóstico, cerca de $61,29 \%$ cursavam com leucocitose e outros 16,13\% com leucopenia, já linfocitose estava presente em $67,74 \%$ das crianças. Em $25,80 \%$ dos pacientes encontrou-se bastonetes aumentados e $58,06 \%$ linfócitos atípicos, além disso, foram encontrados $54,83 \%$ de blastos nos hemogramas e $83,87 \%$ de plaquetopenia. Conclusão: $O$ hemograma demostrou ser um instrumento complementar valioso que associado ao quadro clínico e à perspicácia do médico, pode contribuir para diagnosticar a doença em estágios iniciais, proporcionando tratamento precoce e melhora do prognóstico.

Palavras-Chave: Leucemia linfoide, Contagem de células sanguíneas, Leucemia mieloide.

\begin{abstract}
Objective: To analyze the signs, symptoms and values of the initial blood count of children who later had a definitive diagnosis, using myelogram, of leukemia. Methods: Medical records of 31 individuals, of both sexes, under the age of 18 years, with a diagnosis of lymphoid and myeloid leukemia were evaluated in the oncology service of a hospital in a city in the state of São Paulo. They were evaluated as absolute and determined frequencies, mean, standard deviation, minimum, maximum and average values. The study was approved by the Research Ethics Committee. Results: Considering the blood count variables of the patients analyzed, it is observed that $80.64 \%$ had anemia at the time of diagnosis, about $61.29 \%$ had leukocytosis and another $16.13 \%$ with leukopenia, while lymphocytosis was present in $67.74 \%$ of children. In $25.80 \%$ of the patients, enlarged rods and $58.06 \%$ atypical lymphocytes were found, in addition, $54.83 \%$ of blasts were found in the hemograms and $83.87 \%$ of thrombocytopenia. Conclusion: The blood count has proven to be a valuable complementary instrument that, associated with the clinical condition and the physician expertise, can contribute to diagnose the disease in early stages, providing early treatment and improving the prognosis.
\end{abstract}

Key words: Leukemia lymphoid, Blood cell count, Leukemia myeloid.

\section{RESUMEN}

Objetivo: Analizar los signos, síntomas y valores del recuento sanguíneo inicial de niños que luego tuvieron un diagnóstico definitivo, a través de mielografía, de leucemia. Métodos: Los registros médicos de 31 individuos, de ambos sexos, menores de 18 años, con diagnóstico de leucemia linfoide y mieloide fueron evaluados en el servicio de oncología de un hospital en una ciudad del estado de São Paulo. Se evaluaron como frecuencias absolutas y determinadas, media, desviación estándar, valores mínimos, máximos y medios. El estudio fue aprobado por el Comité de Ética en Investigación. Resultados: Teniendo en cuenta las variables de recuento sanguíneo de los pacientes analizados, se observa que el $80.64 \%$ tenía anemia al momento del diagnóstico, aproximadamente el $61.29 \%$ tenía leucocitosis y otro $16.13 \%$ con leucopenia,

${ }^{1}$ Faculdade de Medicina da Universidade de Franca (UNIFRAN), Franca - São Paulo. *E-mail: robsondutra50@gmail.com 
mientras que la linfocitosis estaba presente en $67.74 \%$ de los niños. En el $25.80 \%$ de los pacientes, se encontraron varillas agrandadas y $58.06 \%$ de linfocitos atípicos, además, el $54.83 \%$ de los blastos se encontraron en los hemogramas y el $83.87 \%$ de la trombocitopenia. Conclusión: El recuento sanguíneo demostró ser un valioso instrumento complementario que, asociado con la condición clínica y la perspicacia del médico, puede contribuir a diagnosticar la enfermedad en etapas tempranas, proporcionando un tratamiento temprano y mejorando el pronóstico.

Palabras clave: Leucemia linfoide, Recuento de células sanguíneas, Leucemia mieloide.

\section{INTRODUÇÃO}

A incidência de crianças com diagnóstico de câncer aumentou de 11,5 para 14,8 casos por 100.000 crianças, sendo a leucemia o mais comum tipo de câncer infanto-juvenil (cerca 35\% dos cânceres pediátricos) $(\mathrm{NCl}, 2012)$. Apesar desse aumento na incidência de câncer na infância, tem-se observado, um aumento das taxas de cura dessas neoplasias. Os avanços na terapêutica e os métodos de detecção precoce têm possibilitado, sobrevida de mais de 70\% quando o diagnóstico é precoce (INCA, 2016).

A leucemia é oriunda das células hematopoiéticas possuindo, portanto, início na medula-óssea e posteriormente invade o sangue periférico, podendo atingir vários órgãos do paciente. Existem quatro tipos principais de leucemias: Leucemia Linfocítica Aguda (LLA); Leucemia Mielocítica Aguda (LMA); Leucemia Linfocítica Crônica (LLC); Leucemia Mielocítica Crônica (LMC) (HAMERSCHLAK N, 2008).

A LLA, especialmente em crianças abaixo dos 5 anos, é a mais comum, representando cerca de $80 \%$ de todas as leucemias nessa faixa etária (INCA, 2017). Ela é derivada de várias alterações genéticas que ocorrem nas células sanguíneas progenitoras que darão origem à célula $T$ ou $B$, incluindo mutações que permitem às células capacidade ilimitada de auto-renovação. Além do acúmulo de alterações na estrutura ou expressão de certos genes vitais, outros fatores podem influenciar a susceptibilidade individual, tais como grupo étnico, idade, sexo e infecções virais (PUI CH, 2008).

A LMC é uma doença mieloproliferativa crônica clonal, caracterizada pela presença do cromossomo Philadelphia $(\mathrm{Ph})$, resultado de uma translocação balanceada entre os braços longos do cromossomo 9 e 22 , levando à fusão dos genes BRC e ABL. Esse novo rearranjo leva à formação de uma nova proteína, com atividade tirosinoquinase desregulada. A doença evolui em três fases: crônica, acelerada e crise blástica. $O$ quadro clínico na fase inicial da doença, pode apresentar parestesia palmar, hepatoesplenomegalia, leucocitose com desvio à esquerda, além de sintomas de hipercatabolismo, como fadiga, perda ponderal, sudorese noturna e febre (LEE MLM, el al., 2008; FERMO VC, 2014).

A LMC é rara na infância, representando apenas $2 \%$ a 3\% das leucemias em crianças e adolescentes menores de 15 anos, mais de $80 \%$ dos casos são diagnosticados após os 4 anos de idade, sendo observado que mais de 60\% desses ocorrerão em crianças maiores de 10 anos (FERMO VC, 2014).

Em crianças menores de 10 anos, a LMA representa cerca de 10\% das leucemias aumentando a incidência com a evolução da idade. Ocasiona falência medular e infiltração orgânica de blastos, o que leva desde anemia até sangramentos graves. Já a LLC é a leucemia que acomete mais adulto, com diagnóstico médio aos 70 anos, sendo uma neoplasia maligna constituída por um acúmulo progressivo de linfócitos maduros incompetentes quanto a funcionalidade de origem clonal (GARNICA M, 2005; HELMAN R, 2011).

Os principais fatores de risco da leucemia, segundo a Associação Brasileira de Hematologia, Hemoterapia e Terapia Celular $(\mathrm{ABHH})$ são principalmente: a exposição a altas doses de radioatividade e produtos químicos como benzeno e pesticidas. Os fatores ambientais como alimentação e poluição atmosférica exercem pouca influência na carcinogênese dos tumores pediátricos, o que dificulta a prevenção primária (SOSSELA FR, 2003).

A morbimortalidade das crianças e adolescentes com câncer é influenciada diretamente pela precocidade do diagnóstico. Embora a taxa de cura do câncer infanto juvenil nos países desenvolvidos supere $70 \%$, no Brasil, dados oficiais mostram que o país está aquém desse percentual. Pode-se atribuir essa defasagem à 
demora na suspeita do diagnóstico que, caso fosse realizado precocemente, agilizaria a referência desses pacientes à atenção especializada (INCA, 2013).

As manifestações clínicas das leucemias linfocítica e mieloide são secundárias à proliferação excessiva de células imaturas (blásticas) da medula óssea, as quais infiltram os tecidos do organismo, tais como: amígdalas, linfonodos, pele, baço, rins, sistema nervoso central e outros (MINISTÉRIO DA SAÚDE; 2017; PEDROSA F, et al., 2002). Com a evolução da doença, os sinais e sintomas clínicos refletem a falência medular resultando em anemia, neutropenia e plaquetopenia, levando à palidez, petéquias, equimoses e eventuais sangramentos (LEE MLM, el al., 2008; FERMO VC, 2014).

O hemograma é o primeiro passo para a investigação laboratorial do paciente com sintomas que possam gerar a suspeita de uma leucemia. Este simples exame expressa as condições reativas do sangue periférico, e deve ser interpretado a luz dos sinais e sintomas (ANDRIOLO A, et al., 2008). A presença de neutrófilos não segmentados (bastão, metamielócito, mielócito e promielócito), linfócitos variantes (linfócitos atípicos, células linfomatosas) e blastos (células imaturas leucêmicas) são identificados pelos métodos automatizados e um alerta correspondente é gerado pelo analisador hematológico (ANDRIOLO A, et al., 2008).

Aproximadamente metade dos casos tem nível de hemoglobina abaixo de $8 \mathrm{~g} / \mathrm{dL}$, embora possam ocorrer valores normais. A anemia é, em geral, normocítica e normocrômica, observando-se às vezes alterações morfológicas como dacriócitos e presença de eritroblastos. O número de leucócitos pode ser normal, aumentado ou mesmo diminuído (MILLER O, et al., 1999).

Aproximadamente $40 \%$ dos pacientes têm níveis inferiores a $10.000 / \mathrm{mm}$, mas podem atingir números superiores a 100.000/mm. Desta forma, as alterações encontradas no hemograma, devem influenciar no seguimento da investigação, possibilitando um diagnóstico mais precoce (SOSSELA FR, et al., 2017).

No diagnóstico de LMC, deve-se solicitar o hemograma completo, mielograma, biópsia de medula com coloração pela prata (para avaliar a presença de fibrose), cariótipo da medula óssea (presença do cromossomo Philadelphia confirma o diagnóstico), Reação de Cadeia de Polimerase (PCR) qualitativo (para identificação do transcrito BCR-ABL) e PCR quantitativo, ambos para diagnóstico e acompanhamento. O Hibridização Fluorescente in situ (FISH) é outro tipo de exame que avalia os cromossomos e detecta as alterações pequenas não visualizadas (SOSSELA FR, et al., 2017).

A maioria dos antígenos pesquisados tem marcadores expressos principalmente na membrana e, por vezes, no citoplasma e no núcleo, podendo ser detectados pelos anticorpos monoclonais através da citometria de fluxo. Os pacientes com LMC possuem a medula óssea com hipercelularidade devido a hiperplasia mieloide, com células em diferentes fases de maturação e diminuição da série vermelha. Observa-se, também, a proliferação de megacariócitos, graus variáveis de fibrose reticulínica e vascularização. A confirmação do diagnóstico deve sempre ser feita com a imunofenotipagem, técnica que permite não só identificar a linhagem, mas também a fase de diferenciação celular (USP, 2010).

A LLA pode ser classificada, de acordo com o imunofenótipo, como derivada de precursores de células $B$ em torno de $70 \%$ dos casos, precursores de células $T$ em $15 \%$ e de células B e T em menos de $5 \%$. Crianças com idade inferior a 18 meses ou maiores que dez anos igualmente têm pior prognóstico (USP, 2010).

O tratamento completo das LA deve considerar a imunofenotipagem, a citogenética, a contagem inicial de glóbulos, as condições clínicas e o envolvimento ou não do sistema nervoso central, testículos e gânglios (MINISTÉRIO DA SAÚDE, 2017; PEDROSA F, et al., 2002). O tratamento da LLA é prolongado, variando de dois a três anos. Os protocolos são constituídos de cinco grandes fases: indução da remissão, intensificaçãoconsolidação, reindução, prevenção da leucemia no sistema nervoso central e continuação ou manutenção da remissão (PEDROSA F, et al., 2002).

$\mathrm{Na}$ LMC, o tratamento pode ser medicamentoso, que tem como primeira linha o Mesilato de Imatinibe (inibidor da tirosina-quinase) ou ser feito por meio do alotransplante de células progenitora hematopoiéticas (terapia celular com efeito curativo) que é recomendado para pacientes jovens e quando apresentarem resistência ou reposta insatisfatória ao tratamento medicamentoso. A decisão do transplante de medula óssea 
passa a ser baseada em três principais variáveis: idade do paciente, grau de resposta ao imatinibe e a disponibilidade de doador (LEE MLM, el al., 2008; SOSSELA FR, et al., 2017). As leucemias são altamente prevalentes e estão associadas com uma significativa morbimortalidade infantil. O hemograma constitui um exame laboratorial que pode revelar grandes indícios da presença dessa patologia, levando a um diagnóstico precoce, fato que aumenta as chances de cura (INCA, 2013).

O câncer não será a primeira hipótese considerada pelo médico diante de queixas inespecíficas. Entretanto, de acordo com o Ministério da Saúde, qualquer criança que se apresente com os sintomas de sangramento anormal, febre, fadiga, palidez, dor óssea, hepatoesplenomegalia e linfadenomegalia generalizada, deve ter um hemograma solicitado. A não suposição de se tratar de uma doença grave em pacientes oligossintomáticos pode retardar o diagnóstico (PEDROSA F, et al., 2002). A relevância deste estudo se dá pela necessidade premente de alertar e auxiliar os profissionais médicos para um diagnóstico presuntivo das leucemias, com vistas a melhorar os desfechos em saúde para essa população específica.

Diante disso, o nosso objetivo foi de descrever os sinais, sintomas e os valores do hemograma inicial de crianças diagnosticadas com leucemia por meio de estatística descritiva, testar associações bivariadas, caso elas existam, entre dados clínicos e os dados do hemograma de crianças diagnosticadas com leucemia, além de investigar correlações entre os achados do hemograma desses pacientes com o diagnóstico médico final.

\section{MÉTODOS}

Estudo retrospectivo analítico e transversal com análise quantitativa dos dados. O critério de inclusão no estudo foram crianças com mais de 6 meses e adolescentes com até 18 anos de idade admitidos no serviço de Oncologia de um Hospital em uma cidade do estado de São Paulo com o diagnóstico de leucemia no período do estudo, excluindo todos pacientes sem diagnóstico confirmado e os que não possuíam hemograma inicial.

Os dados obtidos incluem variáveis sociodemográficas (idade, sexo, cor, naturalidade), dados clínicos (primeiros sinais e sintomas) e relacionados ao hemograma (série branca, série vermelha e plaquetas). Foram avaliados os prontuários de 31 indivíduos com diagnóstico de LLA e LMA, após a aprovação do Comitê de Ética em Pesquisa (parecer de no 1.095.700). O hemograma analisado foi o primeiro realizado pelo paciente durante a investigação da leucemia antes da confirmação diagnóstica pelo mielograma.

Os valores de referência utilizados no hemograma foram divididos pela faixa etária de 6 meses à 2 anos: Hemoglobina 10,5 a $12 \mathrm{~g} / \mathrm{dL}$; Hemácias 3,7 a 4,5 mi/mm3, Leucócitos 6 a 17 milhares $/ \mathrm{mm} 3$, Neutrófilos 1500 a $8500 / \mathrm{mm} 3$, Segmentados 33 a 55\%, Plaquetas 200.000 a 400.000/mm3, Linfócitos 2 a 8 milhares $/ \mathrm{mm} 3$, Blastos ausentes;

3 anos aos 6 anos: Hemoglobina 11,5 a 12,5 g/dL; Hemácias 3,9 a 4,6 milhões/mm3, Leucócitos 5000 a $14500 / \mathrm{mm} 3$, Neutrófilos 1500 a $8000 / \mathrm{mm} 3$, Segmentados 33 a $55 \%$, Plaquetas 150.000 a $450.000 / \mathrm{mm} 3$, Linfócitos 1500 a 7000/mm3, Blastos ausentes;

7 anos aos 13 anos: Hemoglobina 11,5 a 13,5 g/dL; Hemácias 4 a 4,6 milhões/mm3, Leucócitos 5000 a $13000 / \mathrm{mm} 3$, Neutrófilos 1800 a 8000/mm3, Bastonetes 1 a $5 \%$, Segmentados 33 a 55\%, Plaquetas 150.000 a 450.000/mm3, Linfócitos 1500 a 7000/mm3, Blastos ausentes;

acima de 14 anos: Hemoglobina 11,5 a 12,5 g/dL; Hemácias 3,9 a 4,6 milhões/mm3, Leucócitos 5000 a $14500 / \mathrm{mm} 3$, Neutrófilos 1500 a 8000/mm3, Bastonetes 1 a $5 \%$, Segmentados 33 a 55\%, Plaquetas 150.000 a 450.000/mm3, Linfócitos 1000 a 5000 milhões $/ \mathrm{mm} 3$, Blastos ausentes.

Será considerado a presença de sangramento ativo quando houver petéquias e/ou epistaxe, plaquetopenia (menor do que $20.000 / \mathrm{mm}$ ), leucocitose (maior que 50.000/mm) e anemia grave (hemoglobina menor que 6,0 $\mathrm{g} / \mathrm{dL})$.

Foram avaliadas as frequências absolutas e relativas, média, desvio padrão, valores mínimos, máximos e mediana.

REAS/EJCH | Vol.12(7) | e3529 | DOI: https://doi.org/10.25248/reas.e3529.2020 Página 4 de 8 


\section{RESULTADOS}

A leucemia linfoide aguda foi o diagnóstico final encontrado em $74 \%$ dos prontuários, $26 \%$ dos pacientes apresentaram diagnóstico de leucemia mieloide aguda. A maioria dos pacientes era do sexo feminino (55\%) e residiam em Franca (74\%). Em relação à cor, $71 \%$ dos pacientes eram brancos e $6,5 \%$ eram negros. Durante o tratamento, 12 (38,5\%) pacientes foram a óbito devido à gravidade do quadro (Tabela 1).

Tabela 1 - Prevalência por sexo, raça, diagnóstico e evolução, n=31, 2018.

\begin{tabular}{lll}
\hline Características Demográficas & N & $\%$ \\
\hline Sexo & & \\
\hline Masculino & 14 & 45 \\
Feminino & 17 & 55 \\
\hline Cor & & \\
\hline Branca & 22 & 71 \\
Negra & 2 & 6,5 \\
Sem cor especificada & 7 & 22,5 \\
\hline Diagnóstico & & \\
\hline Leucemia Linfocítica Aguda & 23 & 74 \\
$\quad$ Leucemia Mielóide Aguda & 8 & 26 \\
\hline Evolução & & \\
\hline Continuou tratamento & 19 & 61,5 \\
$\quad$ Óbito & 12 & 38,5 \\
\hline
\end{tabular}

Fonte: Dutra RA, et al., 2018.

Os sinais e sintomas mais frequentes na primeira consulta foram: febre $(48,5 \%)$; dor em membros superiores/inferiores (29\%); dor abdominal (26\%); episódios de sangramento (19,5\%), linfadenopatia (13\%), palidez (16\%) e aumento do volume abdominal (9,5\%) (Tabela 2).

Tabela 2 - Primeiros sinais e sintomas apresentados pelos pacientes, $n=31.2018$.

\begin{tabular}{lll}
\hline Sinais e sintomas & $\mathbf{N}$ & $\%$ \\
\hline Febre & 15 & 48,5 \\
Dor musculoesquelética & 9 & 29 \\
Dor abdominal & 8 & 26 \\
Linfadenopatia & 4 & 13 \\
Aumento do volume abdominal & 3 & 9,5 \\
Palidez & 5 & 16 \\
Episódios de sangramento (epistaxe/equimoses) & 6 & 19,5
\end{tabular}

Fonte: Dutra RA, et al., 2018.

Considerando-se as variáveis dos hemogramas dos pacientes analisados, observamos que $80,64 \%$ apresentavam anemia; apenas $9,7 \%$ continham valores de hemoglobina dentro da normalidade. Cerca de $61,29 \%$ cursavam com leucocitose e outros $16,13 \%$ com leucopenia. Linfocitose foi encontrada numa faixa de $67,74 \%$. Linfopenia foi encontrado em $6,46 \%$, Linfócitos atípicos também foi um achado da maior parte 
dos pacientes (58,06\%); somente 6,45\% possuía valores de mielócitos presentes e 9,67\% metamielócitos presentes. Blastos foram encontrados em $54,83 \%$ dos pacientes neste primeiro hemograma, enquanto em $45,17 \%$ estavam ausentes. A grande maioria $(83,87 \%)$ apresentavam plaquetopenia no hemograma inicial, enquanto apenas $26,4 \%$ dos exames continha valores dentro da normalidade (Tabela 3 ).

Tabela 3 - Resultados das variáveis do HMG (hemograma), 2018.

\begin{tabular}{lll}
\hline Alteração & Total de pacientes & $\begin{array}{l}\text { Frequência de } \\
\text { ocorrência }\end{array}$ \\
\hline Anemia & 25 & $80,64 \%$ \\
Plaquetopenia & 26 & $83,87 \%$ \\
Leucocitose & 19 & $61,29 \%$ \\
Leucopenia & 5 & $16,13 \%$ \\
Linfocitose & 21 & $67,74 \%$ \\
Linfopenia & 2 & $6,46 \%$ \\
Linfócitos atípicos & 18 & $58,06 \%$ \\
Bastonetes aumentados & 8 & $25,80 \%$ \\
Mielócitos presentes & 2 & $6,45 \%$ \\
Metamielócitos presentes & 3 & $9,67 \%$ \\
Blastos presentes & 17 & $54,83 \%$ \\
\hline
\end{tabular}

Fonte: Dutra RA, et al., 2018.

\section{DISCUSSÃO}

A leucemia é a principal neoplasia que acomete as crianças e adolescentes, possuindo um período de latência curto com o surgimento dos sintomas em poucas semanas. Nossos resultados demonstraram 0 predomínio da LLA, totalizando 23 pacientes (74\%); sete (26\%) crianças foram diagnosticadas com LMA e apenas um caso com o diagnóstico de LMC que foi excluído da análise por não possuir hemograma inicial. Tais dados corroboram com a literatura, na qual a LLA é a mais frequente, correspondendo de $75 \%$ a $80 \%$ dos casos, seguida pela LMA, entre 15\% e 20\%. A LMC é incomum, variando de 2 a 5\% na infância e adolescência (INCA, 2017; ASSOCIAÇÃO BRASILEIRA DE LINFOMA E LEUCEMIA, 2016). Neste estudo, o óbito ocorreu em $38,5 \%$ do total de pacientes, (12 crianças), enquanto $19(61,5 \%)$ permaneceram vivas, sendo que a leucemia é uma condição grave, maligna e incidente, associada a elevada letalidade.

Os sintomas das neoplasias malignas da criança são vagos em sua fase inicial, portanto é essencial obter uma história completa do curso da doença. A história patológica pregressa também é importante, pois uma criança com câncer prévio tem maior chance de desenvolver um segundo episódio. Da mesma maneira, uma história familiar positiva pode ser fator de risco (GARCEZ ACC, et al., 2010).

Em nosso estudo, muitos desses sinais e sintomas, como palidez, petéquias, equimoses, febre, dor óssea e linfadenomegalia, estavam presentes na primeira consulta. A febre na criança é um dos sinais mais comuns que motivam a busca pelo atendimento primário, sendo a queixa principal em $30 \%$ das visitas aos consultórios pediátricos. Pode ocorrer em cerca de $50 \%$ a $60 \%$ dos casos de leucemia, sendo uma manifestação relacionada à produção de citocinas. Frequentemente, pode estar associada à infecção bacteriana secundária à neutropenia. (NATIONAL CANCER INSTITUTE, 2012). Em um estudo do Hospital do Câncer de São Paulo com crianças com leucemia, a febre foi a queixa inicial mais frequente, seguida por adenomegalias, equimoses e palidez (CAMPOS LMA, et al., 2008). Resultado semelhante foi encontrado em nosso estudo. Os sintomas musculoesqueléticos podem ser a manifestação inicial das leucemias em crianças. As articulações dos joelhos são as mais acometidas, porém articulações de tornozelos, punhos, cotovelos e quadris também são descritas (GARCEZ ACC, et al., 2010). 
O Ministério da Saúde aponta o hemograma como um instrumento importante na investigação diagnóstica das leucemias. Na presença de um ou mais desses sinais e sintomas citados a seguir, faz-se necessária a realização de um hemograma com liberação do resultado em 24 horas: palidez cutâneo-mucosa, fadiga, irritabilidade, sangramentos anormais sem causa definida, febre, dor óssea ou articular generalizada, hepatoesplenomegalia, linfadenomegalia generalizada e sinais decorrentes da trombocitopenia, tais como epistaxe, hemorragias conjuntivais, sangramentos gengivais, petéquias e equimoses (MINISTÉRIO DA SÁUDE, 2017).

A dor óssea pode ser um sintoma das doenças mieloproliferativas, geralmente associado a metáfise dos ossos longos. Essa dor da lesão neoplásica normalmente é desproporcional aos achados do exame físico, pode ser caracterizada com início intermitente e evolução para persistente e aumento da intensidade. Dessa forma, a dor óssea merece uma investigação para doenças neoplásicas. Em nosso estudo, a dor nos membros inferiores esteve presente como sintoma inicial em $29 \%$ dos pacientes. Foi publicado em 2008 um estudo investigando a associação entre manifestações musculoesqueléticas e neoplasias em crianças, esse estudo observou-se que em $80 \%$ dos pacientes avaliados, houve relato de dor em membros, prevalecendo nos tornozelos e joelhos, além de formas de artralgia e atrite, desses 52\% tiveram diagnostico final de LLA. Notou-se a relevância de monitorar a evolução dos hemogramas, ao observar que no inicio da investigação diagnóstica (quando o paciente relata dores musculoesqueléticas) a hemoglobina, leucócitos e plaquetas estavam próximos dos níveis de normalidade, já quando ocorre a confirmação diagnóstica de LLA, esses parâmetros do hemograma possuíam alterações (CAMPOS LMA, et al., 2008).

Diante da suspeita de leucemia, são consideradas situações de risco, as quais indicam um encaminhamento rápido, em poucas horas, para tratamento emergencial: sangramento ativo (petéquias, epistaxe), plaquetopenia, leucocitose e anemia grave (MINISTÉRIO DA SAÚDE, 2017). Apenas um caso de plaquetopenia de $17.000 / \mathrm{mm}$ foi observado. Não ocorreram casos de leucocitose acima de 50.000 e três pacientes apresentaram anemia grave.

No hemograma usado na investigação laboratorial inicial de dores muscoloesqueléticas em crianças com LLA, outro estudo encontrou alterações em $64 \%$ dos pacientes, sendo $48 \%$ com anemia (com hemoglobina menor que $10 \mathrm{~g} / \mathrm{dl}$ ), em $16 \%$ leucopenia (menor que $4.500 / \mu \mathrm{l}$ ), em $12 \%$ leucocitose (maior que $15.000 / \mu \mathrm{l}$ ), em $20 \%$ plaquetopenia (menor que $150.000 / \mu \mathrm{l}$ ) em cinco (20\%) e em $12 \%$ plaquetose (maior que $500.000 / \mu \mathrm{l}$ ) e presença de Blastos na periferia em $8 \%$ ),em nosso estudo, blastos foram encontrados em $54,83 \%$ e plaquetopenia em $83,87 \%$ dos hemogramas, considerando que pode ocorrer plaquetopenia em cerca de $80 \%$ dos casos de leucemias agudas os dados desse estudo estão de acordo com o outro estudo analisado e com Ministério da Saúde (Garcez ACC, et al., 2010; MINISTÉRIO DA SAÚDE, 2017).

Em um estudo realizado na cidade de São Paulo, crianças e adolescentes com leucemia, cerca de $43 \%$ delas tinham leucócitos abaixo de 10.000/mm3 e 60\% tinham hemoglobina abaixo de $8 \mathrm{mg} / \mathrm{dL}$ (RODRIGUES $\mathrm{KE}$, et al., 2003). No presente estudo, no primeiro hemograma, a minoria dos pacientes $(20,4 \%)$ apresentava valores normais de leucócitos totais. Valores de hemoglobina abaixo de $8 \mathrm{mg} / \mathrm{dL}$ foram encontrados em $20 \%$ dos pacientes.

Ressaltamos a escassa literatura envolvendo a análise dos hemogramas iniciais de pacientes diagnosticados com leucemias, o que indica a necessidade de mais estudos abordando esse tema, inclusive com populações maiores, para que se possa obter parâmetros mais fidedignos de comparação, tendo em vista as múltiplas formas de manifestação de tal patologia.

\section{CONCLUSÃO}

As primeiras manifestações das crianças com leucemia foram sinais e sintomas inespecíficos como: febre, dor em membros inferiores, anemia, leucocitose/leucopenia e plaquetopenia. O hemograma se mostrou ser um instrumento complementar valioso, de fácil acesso, que aliado ao quadro clínico do paciente e à perspicácia do profissional médico, pode contribuir para identificar a doença em estágios iniciais e proporcionar um rápido início do tratamento com um melhor prognóstico. 


\section{AGRADECIMENTOS E FINANCIAMENTO}

Agradecemos ao serviço de Oncologia do Hospital.

\section{REFERÊNCIAS}

1. ANDRIOLO A. Medicina laboratorial. 2nd ed. Barueri, SP: Manole; 2008,120p

2. ASSOCIAÇÃO BRASILEIRA DE LINFOMA E LEUCEMIA. Câncer infantil - leucemia mieloide crônica (LMC). São Paulo: ABLL, 2016.

3. CAMPOS LMA, et al. Comprometimento musculoesquelético como primeira manifestação de neoplasias. Rev Assoc Med Bras., 2008; 54(2): 132-8.

4. FERMO VC, et al. O diagnóstico precoce do câncer infantojuvenil: o caminho percorrido pelas famílias. Esc Anna Nery, 2014; 18(1): 54-9.

5. GARCEZ ACC, et al. Sintomas inespecíficos no diagnóstico de leucemia na infância. Rev Med Minas Gerais, 2010; 20(2):139-141.

6. GARNICA M, NUCCI M. Epidemiologia, tratamento e profilaxia das infecções na leucemia linfóide crônica. Rev. Bras. Hematol. Hemoter., 2005; 27(4): 209-300.

7. HAMERSCHLAK N. Leucemia: fatores prognósticos e genética. Jornal de Pediatria, 2008; 84 (4): 52-57.

8. HELMAN R. Leucemia mieloide aguda: atualidade brasileira de diagnostico e tratamento. Einstein, 2011; 9(2):17983.

9. IKEUTI OS, et al. Dor óssea e sua relação na apresentação inicial da leucemia linfóide aguda. Rev. Bras. Hematol. Hemoter., 2006; 28(1): 45-48.

10. INSTITUTO NACIONAL DE CÂNCER JOSÉ ALENCAR GOMES DA SILVA. Diagnóstico precoce do câncer na criança e no adolescente. Rio de Janeiro: INCA, 2013.

11. INSTITUTO NACIONAL DE CÂNCER JOSÉ ALENCAR GOMES DA SILVA. Estimativa 2017: Incidência de Câncer no Brasil. Rio de Janeiro: INCA, 2016.

12. INSTITUTO NACIONAL DE CÂNCER JOSÉ ALENCAR GOMES DA SILVA. Incidência, mortalidade e morbidade hospitalar por câncer em crianças, adolescentes e adultos jovens no Brasil: informações dos registros de câncer e do sistema de mortalidade. Rio de Janeiro: INCA, 2017.

13. LEE MLM. Leucemia Mielóide Crônica em pediatria: perspectivas atuais. Rev. Bras. Hematol. Hemoter., 2008; 30(1): 59-65.

14. MILLER O. Laboratório para o clínico. 8nd ed. São Paulo: Editora Atheneu; 1999; 486 p.

15. MINISTÉRIO DA SAÚDE. Protocolo de diagnóstico precoce do câncer pediátrico. Ministério da Saúde, $2017:$ 4-26.

16. NATIONAL CANCER INSTITUTE. Cancer Topics. NCI, 2012.

17. PEDROSA F, LINS M. Leucemia linfoide aguda: uma doença curável. Rev. bras saúde matern. Infant., 2002; 2(1): 63-68.

18. PEREIRA WV. Aspectos epidemiológicos, biotipologia e evolução do tratamento da Leucemia Linfocítica Aguda na Infância e Adolescência no Rio Grande do Sul. Tese (doutorado). São Paulo: Faculdade de Medicina da Universidade de São Paulo - USP; 2010; 340 p.

19. PUI CH, et al. Acute lymphoblastic leukaemia. The Lancet, 2008; 371:1030-43.

20. RODRIGUES KE, CAMARGO B. Diagnóstico precoce do câncer infantil: responsabilidade de todos. Rev Assoc Med Bras., 2003; 49(1): 29-34.

21. SOSSELA FR, et al. Leucemia Mieloide Crônica: aspectos clínicos, diagnóstico e principais alterações observadas no hemograma. Rev. Bras. Análises Clínicas, 2017; 49(2): 127-30. 